W4C-(14)-5

\title{
MODAL ANALYSIS OF PHOTONIC CRYSTAL PLANAR WAVEGUIDES USING A FINITE DIFFERENCE METHOD
}

\author{
Chin-ping Yu and Hung-chun Chang* \\ Graduate Institute of Communication Engineering, National Taiwan University \\ Taipei, Taiwan 106-17, R.O.C. \\ Phone: +886-2-23635251 ext. 513, Fax: +886-2-23638247 \\ E-mail: hcchang@cc.ee.ntu.edu.tw \\ *Also with the Department of Electrical Engineering and the Graduate Institute of Electro-Optical Engineering, \\ National Taiwan University
}

\begin{abstract}
A finite difference solution method is formulated for solving modes propagating on photonic crysta planar waveguides. The photonic crystal is composed of either air columns or dielectric rods and both $T E$ and TM modes are obtained.
\end{abstract}

Photonic crystals and their photonic device applications have been research topics of great interest in recent years. Photonic crystals with line defects offer a new kind of optical waveguides; which can even guide light through sharp bends [1], [2]. We consider straight waveguides formed by two-dimensional (2-D) photonic crystals, called photonic crystal planar waveguides. The photonic crystal is composed of either air columns or dielectric rods. Although in real device applications, the waveguide might be in the slab form, with the slab perpendicular to the axes of the columns or rods, understanding the characteristics of the simpler planar waveguides is essential. Modal characteristics of the photonic crystal waveguide have been most often studied by using the plane-wave expansion method [3] or by the finite-difference time-domain (FDTD) method [1], [4]. Recently, Toyama et al. [5] provided an accurate analysis of such structures involving multilayered periodic arrays of circular dielectric rods based on the lattice sums technique combined with the T-matrix approach for scattering from a cylindrical object. In this paper we present an efficient finite difference solution method for the same problem. Electromagnetic mode solvers based on the finite difference method (FDM) have been one of the popular techniques for analysis of waveguide modes on various optical or dielectric waveguides. For application to photonic crystal planar waveguides, we modify the mode solver for treating the "supercell" that extends one period along the wave propagation direction and several periods in the transverse direction. Periodic boundary conditions are imposed upon the two boundaries separated by one period and absorbing boundaries are considered for the other two. Propagation constants and the mode fields for both TE and TM modes are successfully obtained and are compared with prior results [4], [5]. For the photonic crystal waveguide studied in [4], there is a line defect in the 2-D square lattice of finite air column in GaAs forming the middle slab of the waveguide. Light can be guided in the middle slab by the Bragg reflection (due to the existence of the photonic bandgap (PBG)) as well as by the mechanism of total internal reflection. The waveguides investigated in [5], however, involve 2-D photonic crystals formed by multilayered periodic arrays of circular dielectric rods. Light can be guided in the middle slab, which is air, only by the mechanism of PBG.

We have demonstrated that the finite difference method can be utilized to efficiently analyze photonic crystal planar waveguides. Our numerical results agree very well with those reported in [4] and [5] based on the FDTD method and the T-matrix approach, respectively.

Acknowledgments-This work was supported by the National Science Council of the Republic of China under Grants NSC91-2215-E-002-020 and NSC91-2215-E-002-031.

\section{REFERENCES}

[1] A. Mekis, J. C. Chen, I. Kurland, S. Fan, P. R. Villeneuve, and J. D. Joannopoulos, "High transmission through sharp bends in photonic crystal waveguides," Phys. Rev. Lett., 77, pp. 3787-3790 (1996).

[2] T. Baba, N. Fukaya, and J. Yonekura, "Observation of light propagation in photonic crystal optical waveguides with bends," Electron. Lett., 35, pp. 654-655 (1999).

[3] H. Benistry, "Modal anaiysis of optical guides with two-dimensional photonic band-gap boundaries," J. Appl. Phys., 79, pp. 7483-7492 (1996).

[4] A. Adibi, Y. Xu, R. K. Lee, A. Yariv, and A. Scherer, "Properties of the slab modes in photonic crystal optical waveguides," J. Lightwave Technol., 18, pp. 1554-1564 (2000).

[5] H. Toyama, K. Yasumoto, and H. Jia, "Electromagnetic scattering and guidance by two-dimensional photonic bandgap structures," Paper DB.P.2, XXII ${ }^{\text {th }}$ General Assembly of the International Union of Radio Science, Maastricht, the Netherlands (August 17-24, 2002). 